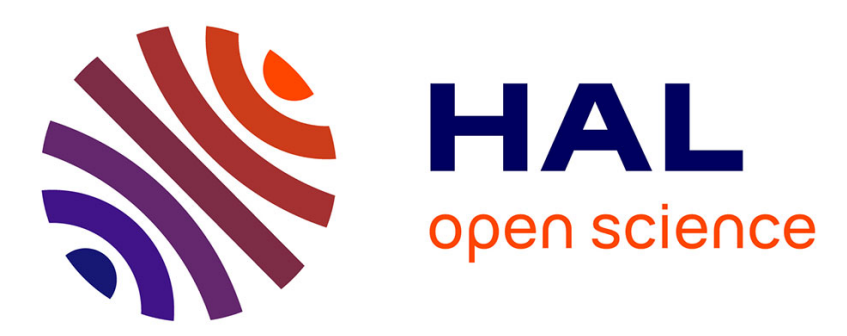

\title{
Space charge effects on interband optical nonlinearities in asymmetric coupled quantum wells
}

\author{
K. Fröjdh, U. Olin, R. Planel
}

\section{To cite this version:}

K. Fröjdh, U. Olin, R. Planel. Space charge effects on interband optical nonlinearities in asymmetric coupled quantum wells. Journal de Physique IV Proceedings, 1993, 03 (C5), pp.C5-233-C5-236. 10.1051/jp4:1993544 . jpa-00251630

\section{HAL Id: jpa-00251630 https://hal.science/jpa-00251630}

Submitted on 1 Jan 1993

HAL is a multi-disciplinary open access archive for the deposit and dissemination of scientific research documents, whether they are published or not. The documents may come from teaching and research institutions in France or abroad, or from public or private research centers.
L'archive ouverte pluridisciplinaire HAL, est destinée au dépôt et à la diffusion de documents scientifiques de niveau recherche, publiés ou non, émanant des établissements d'enseignement et de recherche français ou étrangers, des laboratoires publics ou privés. 


\title{
Space charge effects on interband optical nonlinearities in asymmetric coupled quantum wells
}

\author{
K. FRÖJDH, U. OLIN* and R. PLANEL** \\ Department of Physics II, 10044 Stockholm, Sweden \\ * Institute of Optical Research, 10044 Stockholm, Sweden \\ ** Laboratoire de Microstructures et de Microélectronique, CNRS, 196 avenue Henri Ravera, BP. 107, \\ 92225 Bagneux cedex, France
}

\begin{abstract}
We have studied space-charge-induced optical nonlinearities for asymmetric coupled quantum wells. By the spatial separation of heavy holes and electrons, an electrostatic potential is induced which increases the fundamental bandgap. Calculations, using the Hartree approximation, for optimized AlGaAs structures show that a blue-shift of the band gap of $9 \mathrm{meV}$ at a carrier density of $10^{12} \mathrm{~cm}^{-2}$ can be obtained. Experimental comparisons with a rectangular well show a large increase in room-temperature nonlinearities.
\end{abstract}

\section{Introduction}

Carrier-induced changes of optical properties in semiconductors have potential applications in optical switching and electro-optical modulators. Quantum wells have been extensively studied, mainly due to the saturation of the excitonic absorption at low carrier densities [1]. However, higher carrier densities are needed to get usable nonlinearities for most applications [2]. At those carrier densities other non-linear effects, like bandfilling and bandgap renormalisation, dominate [3]. Unfortunately, these two effects have opposite effects on the absorption. Bandfilling blue-shifts the absorption edge, and bandgap renormalisation produces a red shift. Another contribution to the optical nonlinearities could be obtained by inducing changes of the electrostatic potential, by spatially separating the electrons and the holes. To enhance this electrostatic potential change and increase the nonlinearities, a suitable design of the structure should be chosen. We have investigated asymmetric coupled quantum wells (ACQWs), since for this type of structures a separation of the electrons and holes can be obtained. This system has been studied for a number of other applications, e.g. quantum confined Stark effect [4]-[7], generation of rf oscillations [8] and IR-detection [9]. Optical nonlinearities have previously been studied by Le $e t$ al [10] and Little et al [11] in structures consisting of a wide GaAs well coupled to a narrow $\mathrm{GaAs}$ well under an extrinsic electric field. Dawson et al [12] have studied ACQWs, where the GaAs wells are coupled via the X state in the intermediate AlAs barrier. In this paper room-temperature results are presented for undoped type I structures consisting of a deep narrow well tightly coupled to a wide shallow well, without any extrinsic field.

\section{Self-consistent calculations}

To calculate the contribution from this space-charge effect, the Schrödinger and the Poisson equations have to be solved self-consistently. This is done by coupling the equations in a damped iterative scheme. The Schrödinger equation is solved using the transfer matrix method [13]. The potential is divided into segments, each having a constant potential. As only bound solutions are of interest, the transfer matrix method can be rewritten to use only real-valued matrices and hence the calculations time can be considerably reduced.

The structure is assumed to be intrinsic and the carrier density for holes is identical to the electron density. No external field is applied. The distribution of carriers between subbands is calculated using the Fermi-Dirac distribution and a simple effective in-plane mass model is used for all carriers [14]. The space charge potential 
is found by analytically integrating the probability density multiplied by the carrier density for each subband. The potential of each segment is modified by the averaged space charge potential of the segment. To have a convergent iteration a damped scheme is used. The segment size is chosen to be one monolayer.

\section{Result from calculations}

An asymmetric coupled quantum well has a number of design parameters: the aluminium concentrations of four layers and the widths of three. To simplify the growth of the structure, the aluminium concentration of the deep well was chosen to be zero and the aluminium concentration of the intermediate barrier was set equal to the barrier concentration $x_{b}$. In order to find optimal values for the remaining five parameters $\left(x_{b}\right.$, $x_{w}, d_{n}, d_{b}$ and $\left.d_{w}\right)$, the shift of the transition energy and the squared overlap $\left|\int \psi_{h h_{1}}(z) \psi_{e_{1}}(z) d z\right|^{2}$ between the heavy hole and the electron groundstate as well as the energy difference to the nearest higher transition were studied for a large number of structures at a final carrier density of $10^{12} \mathrm{~cm}^{-2}$. A high value of $x_{b}$ in the barrier was used due to better carrier confinement. Calculations were done for three different widths for the intermediate barrier, $d_{\mathrm{i}}=1 \mathrm{~nm}, 2 \mathrm{~nm}$ and $3 \mathrm{~nm}$. A large width increases the shift but decreases the overlap and $d_{\mathrm{i}}=2 \mathrm{~nm}$ is a good trade-off. The structure is not so sensitive for the width of the wide well $d_{w}$ : an increase of the width enhances the energy shift but decreases the overlap. Here $d_{\mathrm{w}}=7 \mathrm{~nm}$ was chosen as a suitable compromise. The results are shown in Fig 1. For an increasing aluminium concentration of the wide well $x_{w}$ the localisation of the heavy hole ground state change from the wide well to the narrow well, thus giving a large increase in field and energy shift. The electrons are rather delocalized but a decrease of the narrow well width will reduce the electron density in the narrow well thus increasing fields and energy shifts.

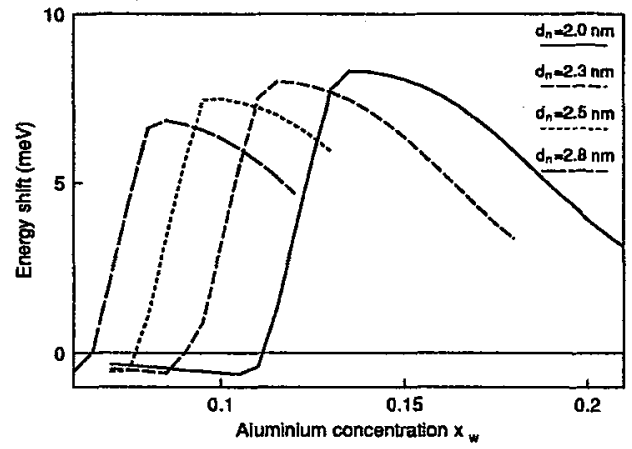

a)

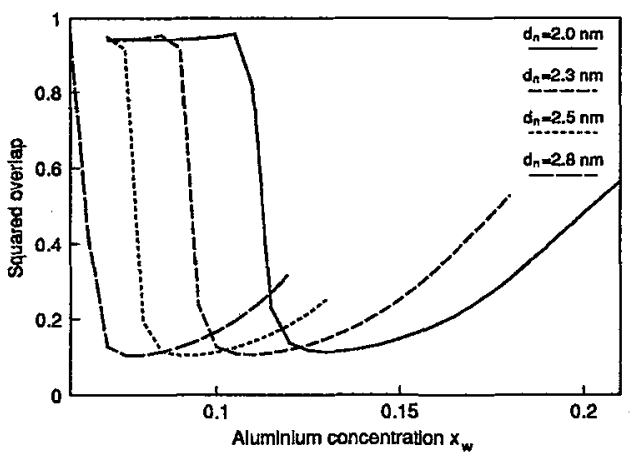

b)

Fig 1. Calculated (a) energy shifts $\Delta \mathrm{E}\left(\mathrm{e}_{1}, \mathrm{hh}_{1}\right)$ and (b) final squared overlap for the heavy hole ground state - electron ground state transition for a sheet carrier density of $10^{12} \mathrm{~cm}^{-2}$. The shift and overlaps are plotted as a function of the aluminium concentration $x_{w}$ of the wide well for different widths $d_{n}$ of the narrow well. 


\section{Experimental procedure and results}

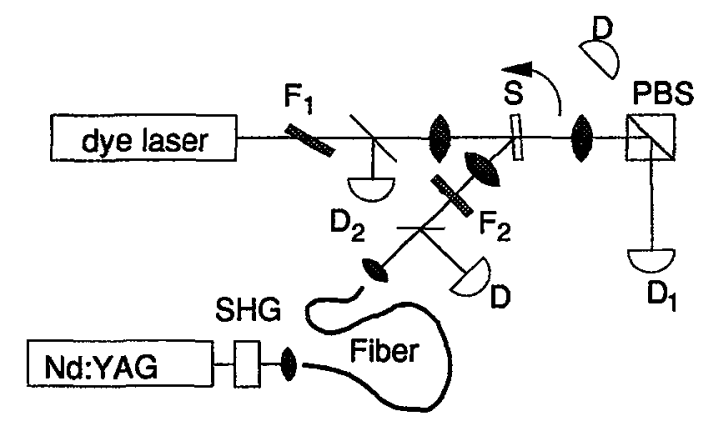

Fig 2. Set-up for measurement of nonlinear absorption

To make an experimental study of this effect a multiple ACQW structure was grown by MBE. The parameters for the structure were chosen such that a large energy shift and a reasonable tolerance for parameter variations. The structure consists of a $2 \mathrm{~nm}$ GaAs narrow well and a $7 \mathrm{~nm}$ thick $A_{10.16} \mathrm{Ga}_{0.84} \mathrm{As}$ wide well, separated by a $2 \mathrm{~nm} \mathrm{Al} \mathrm{Al}_{0.4} \mathrm{Ga}_{0.6} \mathrm{As}$ barrier. For the sake of comparison, a reference sample consisting of $11 \mathrm{~nm}$ thick $\mathrm{Al}_{0.16} \mathrm{Ga}_{0.84} \mathrm{As}$ rectangular $\mathrm{QWs}$ was grown. In both structures $11 \mathrm{~nm} \mathrm{Al}_{0.4} \mathrm{Ga}_{0.6}$ As barriers were used between the wells and the period was repeated 100 times.

The samples were cleaved and cemented to glass slides with the epitaxial side to the glass surface and the substrate was etched away. The non-linear transmission of the wells was measured with a pump-probe set-up shown in Fig 2. The pump beam was generated by a doubled Q-switched Nd:YAG laser giving $200 \mathrm{~ns}$ long pulses at a repetition frequency of $1.2 \mathrm{kHz}$. The pump light was coupled through a multimode fibre, giving a tophat transverse profile. The pump power was varied by inserting neutral density filters $F_{2}$. By replacing the sample with a $50 \mu \mathrm{m}$ pinhole and measuring the transmitted power, the pump irradiance on the sample could be calculated. The probe beam was generated by an argon-ion pumped $C W$ pyridine 2 dye laser. The intensity of the probe was reduced to below $1 \mathrm{~mW}$ with a variable neutral density filter $F_{1}$. The probe was focused on the sample $\mathbf{S}$ and the transmitted light detected with $\mathbf{D}_{1}$. The incident probe power and the transmitted probe power were measured simultaneously with a sampling oscilloscope, thus removing the noise from the dye laser. A polarizing beamsplitter PBS reduced the luminescence background. The residual luminescense background was removed with initial background measurements. The sampling point was adjusted to the peak of the non-linear signal. All measurements were made at room-temperature. The sample could be rotated in a horizontal plane and the transmission was measured for $0^{\circ}$ and $35^{\circ}$ angle of incidence for the TE-polarized probe beam. The Fabry-Perot oscillations of the uncoated sample were reduced by using the geometric mean of these two transmission measurements.

The linear and non-linear of the samples are shown in Fig 3. The linear transmission of the ACQW agrees with the calculated energy levels. The nonlinear transmission shows a large increase of absorption bleaching for the ACQW as compared to the symmetric reference sample. The difference is particularly apparent for small intensities. The total bleaching of the excitonic peaks is clearly visible in the spectra for all carrier densities. In the excited ACQW spectra, a wide spectral region, close to the excitonic peak of the linear spectrum, is bleached. We think this can be attributed do the combined effect of band-filling and space-charge-induced shifts. A saturation of the nonlinearity can be seen for the highest pumping intensity. This can be explained by our calculations. At high carrier density the space-charge induced potential will tend to shift the heavy hole ground state to the wide well, hence removing the spatial separation necessary for the space-charge effect. The absorption shifts $\Delta \alpha$ were Kramer-Kröning transformed to estimate the shift of the refractive index $\Delta n$. The shifts of the refractive index were significantly higher for energies below the bandgap. In conclusion we have shown that carefully designed ACQWs can have significantly enhanced optical nonlinearitites, due to space-charge effects. 


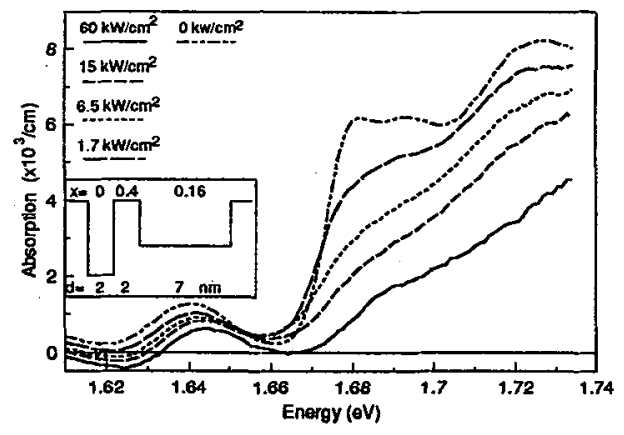

a)

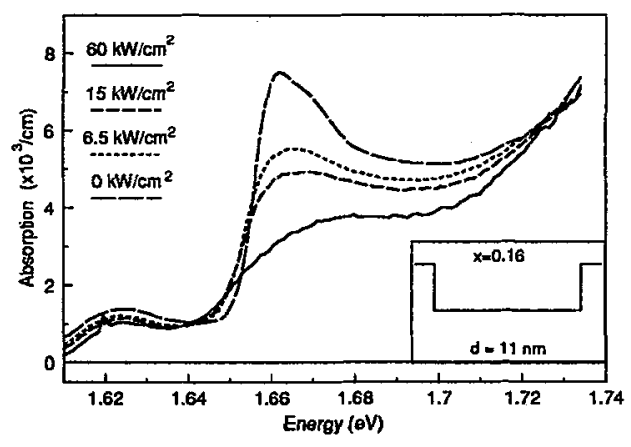

b)

Fig 3. Nonlinear absorption for (a) the ACQW and (b) the reference rectangular QW sample for different pump irradiances. The aluminium concentrations and the well widths for each structure are shown in the insets.

\section{References}

[1] Miller A., Manning R.J., Milsom P. K., Hutching D. C. and Crust D. W., J. Opt. Soc. Am. B. 6 (1989) 567-577.

[2] Milsom P. K. and Miller A., Opt. Quantum Electron. 21 (1989) 81-85.

[3] Schmitt-Rink S., Chemla D. S. and Miller D. A. B., Adv. Phys. 38 (1989) 89-188.

[4] Chan Y. C. and Tada K., IEEE J. Quantum Electron. 27 (1991) 702-707.

[5] Kuhn K. J., Juang C., and Darling R. B., J Appl Phys 69 (1991) 3135-3141.

[6] Juang C., Kuhn K. J. and Darling R. B., IEEE J. Quantum Electron. 27 (1991) 2122-2128.

[7] Ding Y. J., Guo C. L., Khurgin J. B., Law K. K., Stellato J., Law C. T., Kaplan A. E., and Coldren L. A., Appl. Phys. Lett. 59 (1991) 1025-1027.

[8] Luryi S., IEEE J. Quantum Electron. 27 (1991) 54-60.

[9] Vodjdani N., Vinter B., Berger V., Böckenhoff E., and Costard E., Appl. Phys. Lett. 59 (1991) 555-557.

[10] Le H. Q., Hryniewicz J. V., Goodhue W. D. and Mims V. A., Opt. Lett. 13 (1988) 859-861.

[11] Little J. W., Whisnant J. K., Leavitt R. P. and Wilson R. A., Appl. Phys. Lett. 51 (1987) 1786-1788.

[12] Dawson P., Galbraith I., Kucharska A. I. and Foxon C. T., Appl. Phys. Lett. 58 (1991) 2889-2891.

[13] Jonsson B. and Eng S. T., IEEE J. Quantum Electron. 26 (1990) 2025-2035

[14] Bastard G., Wave mechanics applied to semiconductor heterostructures, (les Editions de Physique, Les Ulis, France, 1990), p. 78. 